\title{
Morar en las Lagunas: Lugares de Cazadores Recolectores en la Pampa Bonaerense (Argentina)
}

\author{
Dwelling Next to Lagoons: Places of Hunter-Gatherers in the \\ Buenos Aires Pampas (Argentina)
}

Natalia Mazzia

\begin{abstract}
RESUMEN
El paisaje de los cazadores recolectores resulta un aspecto complejo para estudiar desde la arqueología. En este trabajo se utiliza la noción de lugar arqueológico para abordar la problemática. Su caracterización es posible mediante la integración de diferentes lineas de evidencias que incluyen objetos, espacios y cuerpos. Se analizan las ocupaciones humanas ocurridas durante el Holoceno en la cercanía de las lagunas pampeanas bonaerenses al sur del río Salado (Argentina), con especial énfasis en aquellas descriptas para la localidad arqueológica El Guanaco. A partir de la revisión de distintos ejemplos se propone que los entornos lagunares han sido visitados y habitados, en muchos casos de forma recurrente, a lo largo del Holoceno. La elección de estos espacios involucró, por ejemplo, la planificación de la obtención y el traslado de las materias primas liticas, un escaso aprovechamiento de recursos lacustres $y$, en ciertos casos, el desarrollo de prácticas mortuorias particulares.
\end{abstract}

Las fuentes de agua, por su importancia vital y simbólica, resultan ineludibles en los caminos de los cazadores recolectores. Las aguas quietas de las lagunas pueden relacionarse con momentos de pausa y ésta con los lugares. Por ello, los entornos lagunares representan lugares en la vida de los grupos de cazadores recolectores, quienes, como parte de sus recorridos, volvieron una y otra vez a sus orillas.

Palabras clave: Región Pampeana Bonaerense, Lagunas, Cazadores Recolectores, Paisaje, Lugar

CONICET- Área Arqueología y Antropología, Av. 10 y calle 63 s/nº, Necochea CP 7630, Buenos Aires, Argentina. Correo-e: natymazzia@yahoo.com.ar.

Recibido: 25 de junio 2013 Revisado: 25 de septiembre 2013 Aceptado: 23 de octubre 2013 


\begin{abstract}
The landscape among hunter-gatherers is a complex issue for the archaeological study. Thus, in this paper we introduce the idea of archaeological place as a key concept to address the problem. The characterization of places results from the integration of different lines of evidence including objects, spaces and bodies. The aim of this paper is to discuss human occupations that occurred during the Holocene around the lagoons in Buenos Aires province, south of the Salado River (Argentina), with special emphasis on those evidences described for $E l$ Guanaco locality. Based on the discussion of different examples it is proposed that lagoon environments have been visited and inhabited throughout the Holocene. The choice of these spaces concerned, for example, planning the acquisition and the carrying of lithic raw materials, a low utilization of lake resources and, in some cases, the development of specific mortuary practices.
\end{abstract}

Because of their vital and symbolic importance, water sources are must-visit points along hunter-gatherers pathways. The quiet waters of lagoons may be related to moments of pause, and this pause refers to places. Therefore, lagoons surroundings represent places in the life of hunter-gatherers groups, who, as part of their paths, returned again and again to the shores.

Key words: Pampean Region, Lagoons, Hunter Gatherers, Landscape, Place

\title{
LUGARES Y PAISAJES
}

Los paisajes no son solo lagunas, sierras, arroyos, extensas llanuras o grandes árboles, son también todas aquellas personas que pasaron por ellos. Hombres, mujeres y niños que vivieron experiencias cotidianas en torno a esos espacios, que los recorrieron en busca de refugio, de alimento o de materias primas. Grupos que conocieron cada uno de sus rincones y fueron dejando allí su impronta con el paso del tiempo. Todo espacio se transforma en lugar mediante la intervención humana, los paisajes se van constituyendo como el conjunto de relaciones entre personas y lugares que proporcionan el contexto para la vida diaria (Thomas 2001, Tilley 1994). Ya sea en forma de ríos, arroyos, lagos o lagunas, el agua forma parte de dichos contextos: como recurso indispensable para la vida, como fuente de recursos naturales, creando fronteras, dividiendo u ordenando el espacio, pudiendo incluso resultar un hito sobre el terreno (Richards 1996, Strang 2005). Desde esta perspectiva, el paisaje es entendido como la síntesis de 
la dimensión física del medio natural con la dimensión humana, social y simbólica (Bender 2002, Criado Boado 1999, Curtoni 2007, Ingold 2000, Thomas 2001, Tilley 1994, Troncoso 1999).

La arqueología del paisaje aplicada al estudio de sociedades nómades se encuentra con ciertas limitaciones dictadas por el registro arqueológico. La vida de los grupos de cazadores recolectores suele describirse en base a su alta movilidad, una gran dependencia de los ritmos de la naturaleza para su economía y una importante variabilidad en la densidad poblacional según procesos de agregación y dispersión grupal. Estas características determinan una cierta dificultad al momento de rastrear diferentes componentes que permitan realizar un análisis paisajístico (Diez Martín 2007). Resultan una excepción los estudios que incluyen arte parietal. En ellos, la noción de paisaje socialmente construido se transforma en una herramienta de gran utilidad analítica e interpretativa (por ejemplo: Carden 2008, Curtoni 2007, Mazzanti y Valverde 2003, Taçon, 1994).

Sin registro de arte parietal, la idea de lugar, en tanto espacio vivido e incorporado a las prácticas cotidianas (Thomas 2001), se transforma en un elemento clave a la hora de aproximarse al pasado de este tipo de sociedades desde el enfoque de la arqueología del paisaje considerando su profundidad temporal (Mazzia 2010-2011). En la materialidad de toda práctica el lugar es, a la vez, escenario y objeto, un objeto inmueble que puede ser habitado (Tuan 2008 [1977]). Como entidades físicas, los lugares tienen una locación particular en el espacio. No obstante, simultáneamente están en la mente de las personas, en sus historias y experiencias, en sus costumbres y en sus actividades corporales (Ingold 1993, Low 2003). Los lugares son una pausa en el movimiento (Tuan 2008 [1977]). Los grupos nómades, en sus recorridos constantes a través de un área necesitan decidir dónde establecer sus campamentos, dónde parar por la noche en el medio de algún trayecto. Se trata siempre de elecciones locacionales. En este sentido, los caminos conectan lugares y materializan el movimiento de los cuerpos a través del espacio, permitiendo la creación de un pasado, un presente y un futuro ligados por la experiencia a este movimiento (Potter 2004, Tuan 2008 [1977]). El mundo espacial de los cazadores recolectores nómades puede pensarse como lugares conectados por recorridos.

De esta forma, los paisajes sociales se van conformando por una red de lugares interconectados que se relacionan entre sí mediante las interacciones y las actividades habituales de los grupos humanos, por la proximidad y la afinidad que éstos han desarrollado con esos emplazamientos y por los acontecimientos importantes que hace recordarlos e incorporarlos 
a la memoria (Augé 1998, Thomas 2001). Entonces, una de las formas que tenemos para comprender los paisajes arqueológicos de los cazadores recolectores es conocer cada uno de sus lugares, pensar cómo se relacionan entre sí y cómo cambian o se mantienen a través del tiempo (Mazzia 2013).

En suma, el estudio del detalle en el espacio, definido como la caracterización de lugares arqueológicos, resulta posible gracias a la integración de las diferentes líneas de evidencia, materiales y espaciales, que pueden analizarse en cada sitio. Cada lugar arqueológico es entendido como un nodo dentro de la matriz espacial, conformada por los movimientos de las personas que los conectaron con sus recorridos. Los lugares, las relaciones materiales entre ellos, las prácticas sociales que los enlazan y los caminos que los comunican delinean un entramado en el espacio: los paisajes sociales del pasado. A esto debe sumarse la temporalidad de cada uno de esos lugares, los cambios y las continuidades a lo largo del tiempo que definen la historia y la dinámica de las relaciones entre los grupos humanos y sus paisajes.

En este trabajo se exploran las posibilidades de la arqueología del paisaje en el estudio del pasado de las sociedades de cazadores recolectores mediante una estrategia de investigación centrada en la caracterización de lugares arqueológicos (Mazzia 2013). Se analiza, en particular, el caso de las lagunas pampeanas (provincia de Buenos Aires, Argentina) como entornos visitados y habitados a lo largo del Holoceno. A la luz de dicho análisis, se discute sobre la importancia de las fuentes de agua en la vida de los cazadores recolectores y cómo éstas son incluidas en sus constantes movimientos, transformándose en una pausa, un lugar en su paisaje cotidiano.

\section{LAGUNAS EN LA PAMPA BONAERENSE}

\section{AL SUR DEL RÍO SALADO}

La pampa bonaerense argentina presenta una amplia variedad de ambientes acuáticos, pero con una marcada desproporción entre aguas estancadas y aguas corrientes (Frenguelli 1956). La red fluvial que se origina y circula en este territorio es reducida en relación con la abundancia de espejos de agua, permanentes o temporarios, identificados como lagunas. De acuerdo con Dangavs (2005), la laguna es un cuerpo de agua de profundidad variable, alojado en una cubeta de contorno bien definido. Las lagunas actuales se encuentran en cubetas de distintas edades y origen variado en el que se reconoce la acción de diferentes procesos 
geomorfológicos, pero predominando la deflación, aun en el caso de las depresiones de origen tectónico. El origen y la persistencia de las lagunas están regidos por procesos hidrológicos y meteorológicos: los aportes hídricos provienen de las lluvias, del escurrimiento superficial y del agua libre subterránea (Dangavs 2005).

Las lagunas pampeanas representan espacios en los que se encuentran de forma localizada, gracias a la presencia de agua, una amplia variedad de recursos bióticos. En el área de la depresión del Salado (en el norte de la provincia de Buenos Aires) existe una importante diversidad de sitios arqueológicos que dan cuenta de ocupaciones humanas prolongadas y recurrentes, a partir del Holoceno tardío, en proximidades de cuerpos de agua. En dicha área, las abundantes lagunas, lomadas y bosques de tala definen un ambiente de humedal que representa una importante fuente de aprovisionamiento de alimento, materias primas y combustible (Aldazábal et al. 2004, González et al. 2006). El registro arqueológico que caracteriza a estos sitios presenta ciertas particularidades que lo diferencian de aquel presente en lagunas ubicadas al sur de esta área (por ejemplo: Aldazábal et al. 2004, González 2005, González et al. 2006). La riqueza de la información disponible a partir de las investigaciones realizadas en las proximidades del río Salado excede los límites de la discusión perseguida en este trabajo. Por tal motivo, las evidencias humanas consideradas en torno a los espacios lagunares se restringirán a aquellos sitios arqueológicos ubicados al sur del área de la depresión del Salado. 


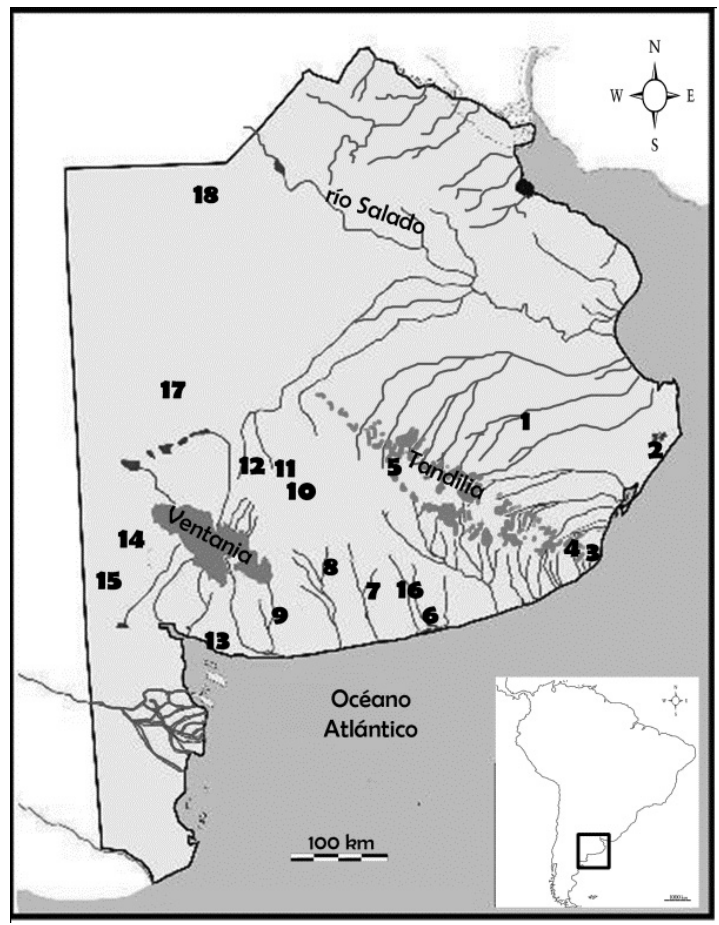

Figura 1: Sitios arqueológicos en cercanías de lagunas: 1- La Colorada; 2-Laguna de Sotelo; 3-Alfar; 4- Cueva La Brava Laguna; 5- La Barrancosa; 6- El Guanaco; 7Arroyo Seco 2; 8-Laguna Tres Reyes; 9-Laguna La Larga; 10-Laguna del Trompa; 11- Fortín Necochea; 12-Laguna La Raquel; 13-Monte Hermoso 1- La Olla 1 y 2; 14-Laguna Los Chilenos; 15-Laguna de Púan; 16-Las Brusquillas; 17-Laguna Arroyo Venado; 18 - Laguna Salalé

Figure 1: Archaeological sites next to lagoons: 1- La Colorada; 2-Laguna de Sotelo; 3Alfar; 4-Cueva La Brava Laguna; 5- La Barrancosa; 6-El Guanaco; 7-Arroyo Seco 2; 8-Laguna Tres Reyes; 9- Laguna La Larga; 10-Laguna del Trompa; 11-Fortín Necochea; 12-Laguna La Raquel; 13-Monte Hermoso 1-La Olla 1 y 2; 14-Laguna Los Chilenos; 15-Laguna de Púan; 16-Las Brusquillas; 17-Laguna Arroyo Venado'; 18-Laguna Salalé

En la llanura interserrana, los sistemas serranos y la costa atlántica de la provincia de Buenos Aires, las lomadas ubicadas en las inmediaciones de los bajos han sido, durante todo el Holoceno, puntos claves del espacio pampeano para los grupos humanos que las visitaron y ocuparon en forma recurrente (Aldazábal et al. 2004, Crivelli Montero et al. 1987/88 a y b, Martínez 1999, Politis y Madrid 2001). Los sitios arqueológicos conocidos en cercanías de lagunas presentan cierta variabilidad en cuanto a su cronología, contexto y funcionalidad; su distribución puede observarse en la Figura 1. Se describirá la información publicada sobre estos sitios pero, previamente, se expondrá el caso de la localidad arqueológica El Guanaco con mayor detalle. 


\section{Localidad arqueológica El Guanaco}

La localidad arqueológica El Guanaco se encuentra en el área interserrana costera, en los alrededores de la laguna El Lucero, a $13 \mathrm{~km}$ de la costa atlántica (Figura 1:6). Esta laguna es un cuerpo de agua semi-permanente y dimensiones medianas, con una superficie aproximada de 35 ha. Allí se han relevado dos sitios arqueológicos y una importante colección de materiales líticos recuperados en superficie ${ }^{2}$. El Guanaco 1 se sitúa en la pendiente de una elevación suave al norte de la laguna. Hacia el este, a unos 500 metros de distancia, se encuentra El Guanaco 2 sobre una pequeña barranca (Figura 2). Las evidencias halladas en ambos sitios dan cuenta de la ocupación humana reiterada de este entorno lagunar a lo largo de todo el Holoceno.

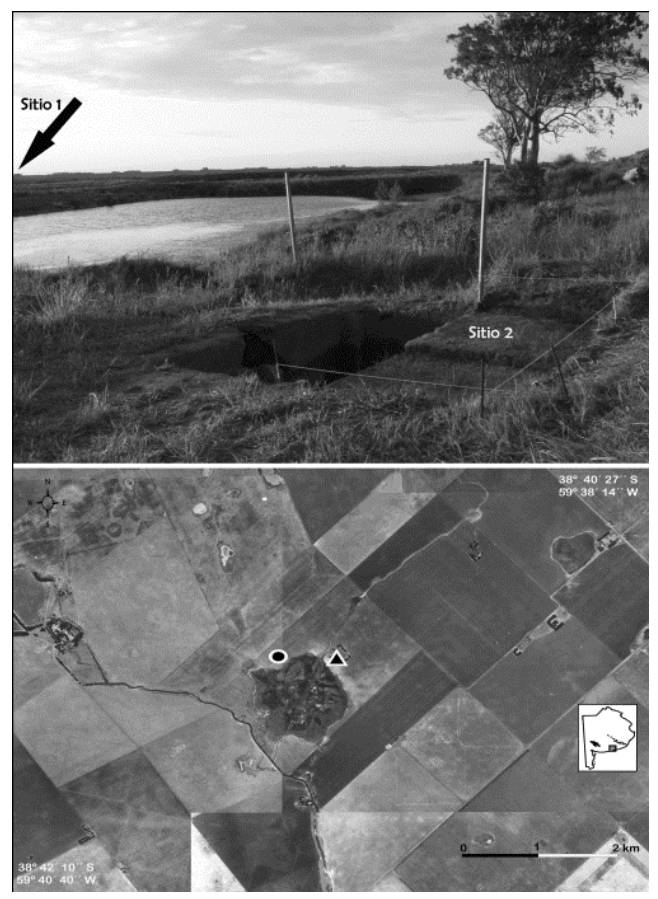

Figura 2: Localidad arqueológica El Guanaco, ubicación de los sitios con respecto al bajo. Referencias: $\mathrm{O}$ - sitio 1; $\triangle$ - sitio 2.

Figure 2: Location of El Guanaco archaeological sites next to the lagoon. References: $\mathrm{O}$ - site $1 ; \triangle$ - site 2.

En el Guanaco 1 se hallaron materiales en toda la secuencia estratigráfica, desde la superficie hasta la tosca de base, característica de estas lomadas cercanas a cuerpos de agua. Esta tosca expuesta, o apenas cubierta por sedimentos, habría sido la superficie del terreno al momento de la llegada de los primeros habitantes durante el Holoceno temprano (Bayón et al. 2004). Por encima de la costra de tosca, arqueológicamente 
estéril, se han recuperado abundantes restos de fauna, entre los que se han podido identificar fragmentos de cáscara de huevo de rheidos, restos óseos de mamíferos extintos: Macrauchenia patachonica y Equus sp., junto a partes esqueletales de fauna autóctona actual. Entre estas últimas se identificaron restos de comadreja colorada, indicador de la proximidad de un cuerpo de agua permanente a comienzos del Holoceno (Bayón et al. 2004). Entre los artefactos líticos tallados se cuentan instrumentos, núcleos y desechos. Este conjunto temprano presenta un amplio predominio de ortocuarcitas del Grupo Sierras Bayas (OGSB) como materia prima, cuyas fuentes de aprovisionamiento se sitúan a más de $100 \mathrm{~km}$ hacia el noreste, en Tandilia (Figura 1; Bayón et al. 2004). Además de los objetos tallados, en este contexto se incluye un molino de mano simple que fue analizado mediante cromatografía gaseosa - espectrometría de masa (CG-EM). A partir de su complementación con el análisis de microfósiles se identificó su utilización para el procesamiento de gramíneas y Celtis tala (Babot et al. 2007).

La ocupación recurrente de este espacio, en el que los grupos humanos llevaron a cabo diferentes actividades de su vida cotidiana y doméstica, se produjo sin mayores cambios desde el Holoceno temprano hasta el Holoceno medio. Con posterioridad, el lugar continuó siendo visitado por diferentes grupos de cazadores recolectores, pero con un objetivo y un sentido diferente. Hace aproximadamente 2500 años AP, en un sector de esta lomada fue cavada una fosa como parte del desarrollo de prácticas mortuorias. La estructura de cavado identificada en el perfil estratigráfico presenta forma de cuenco, mide más de $2 \mathrm{~m}$ de largo y unos $60 \mathrm{~cm}$ de profundidad (Bayón et al. 2004). Debido a la alteración provocada por el arado no resulta posible saber si esta fosa fue demarcada señalando la localización precisa de los entierros. En una superficie de $9 \mathrm{~m}^{2}$ fueron encontrados los restos óseos de siete individuos. La disposición de los cuerpos se organizó en un entierro secundario múltiple y en arreglos de partes esqueletales aisladas. Otros dos individuos fueron inhumados muy cerca de la fosa. Todos estos cuerpos fueron enterrados en un lapso comprendido entre los 2500 y los 2200 años AP. Una última inhumación se produjo hace 1800 años AP repitiendo la modalidad de arreglos de partes esqueletales aisladas (Flegenheimer et al. 2002, Mazzia et al. 2004).

Finalmente, las reocupaciones de esta lomada al norte de la laguna llegan incluso hasta momentos post-conquista. Los materiales correspondientes a este período son escasos, entre ellos se incluyen una cuenta de vidrio y fragmentos de botellas de bebidas alcohólicas, pero sin el registro de restos de especies animales introducidas (Bayón et al. 2004). 
A unos $500 \mathrm{~m}$ hacia el este del sitio 1, otro sector de este entorno lagunar fue ocupado en reiteradas oportunidades durante el Holoceno temprano y medio. El Guanaco 2 se encuentra a pocos metros de una cantera de tosca, actualmente colmada de agua. Los alrededores de esta cárcava fueron limpiados con las mismas maquinarias utilizadas en la cantera, eliminando el horizonte A de suelo. Por este motivo, no se cuenta con evidencias de ocupaciones más tardías de este espacio, en caso de que hayan existido.

Los materiales arqueológicos recuperados se encontraron contenidos en depósitos eólicos que forman un cuerpo medanoso sobre la margen estenoreste de la laguna (Zárate et al. 2009). A diferencia del sitio 1, la tasa de sedimentación en este sector ha sido mayor, por encima de la costra de tosca yacen sedimentos arqueológicamente estériles con fauna extinta. Ésta habría sido la superficie del terreno al momento de la llegada de los primeros habitantes (Zárate et al. 2009). De acuerdo con los resultados de análisis estratigráficos y radiocarbónicos la mayor concentración de evidencias corresponden al lapso comprendido entre los 9000 y los 6500 años AP. En ese período se habrían producido las ocupaciones más densas y/o recurrentes del espacio.

Correspondientes a los momentos más tempranos de ocupación, fueron hallados dos entierros humanos con fechados radiocarbónicos entre los 8500 y 8100 años AP (Flegenheimer et al. 2010). Se trata de un entierro primario de un niño y fragmentos de cráneo de un adulto, cuya forma de inhumación no pudo ser definida. Las prácticas mortuorias no fueron realizadas en un sector destinado únicamente para ese fin, en cambio, se desarrollaron en el mismo espacio en el que tuvieron lugar diferentes tareas relacionadas con la vida doméstica (Flegenheimer et al. 2010). El contexto arqueológico que da cuenta de estas tareas incluye artefactos líticos, pigmentos minerales, restos óseos de fauna autóctona actual, restos malacológicos y cáscaras de huevo de rheidos (Vecchi et al. 2007). El conjunto arqueofaunístico es abundante; a partir de su estudio se identificaron prácticas culinarias llevadas a cabo en el lugar, entre las que se distinguen corte y machacado en los huesos de las especies de porte grande y evidencias de combustión derivadas de la cocción de recursos menores como armadillos y huevos de Rheidae (Frontini 2010, 2012). Los objetos líticos recuperados son principalmente desechos de talla y en menor medida artefactos formatizados y núcleos, entre los que se evidencia una preferencia por la OGSB como materia prima (Vecchi et al. 2007).

En el nivel de ocupación asociado a los fechados del Holoceno medio inicial sobresalen ciertas particularidades, destacándose las evidencias de actividades de molienda y de explotación de rheidos. Los tres artefactos de molienda que forman parte de este contexto fueron analizados mediante CGEM (Babot et al. 2007: 643). En las muestras se detectaron evidencias del 
procesamiento de semillas (Mazzia 2010-2011) y de un posible almacenamiento de los recursos, previo a su molienda. El estudio complementario de los microfósiles indica la presencia de granos de almidón y silicofitolitos de gramíneas y cistolitos de hojas de Celtis tala (Babot et al. 2007).

El otro aspecto particular de las ocupaciones humanas de este espacio registradas a comienzos del Holoceno medio tiene que ver con la presencia de numerosos restos óseos de Rhea americana y la gran cantidad de cáscaras de huevo asignadas a rheidae (Frontini y Picasso 2010).

En suma, El Guanaco 2 representa un lugar en donde, durante el Holoceno temprano, se realizaron entierros humanos en el mismo espacio en donde se llevaron a cabo tareas domésticas relacionadas con la vida cotidiana de los grupos de cazadores recolectores pampeanos. Posteriormente, a comienzos del Holoceno medio, esta lomada arenosa próxima a la laguna continuó siendo un espacio elegido por los grupos humanos que desarrollaron allí prácticas culinarias particulares.

Por último, existeuna importante colección de materialeslíticos quefueron recuperados en superficie en los alrededores de ambos sitios. Estos materiales fueron asignados al Holoceno tardío y evidencian el traslado de un gran volumen de rocas, ya sea en forma de núcleos o artefactos manufacturados, provenientes de ambos sistemas serranos y de la costa atlántica (Bayón y Flegenheimer 1998). La materia prima más empleada para tallar los instrumentos fue nuevamente la OGSB. En esta colección sobresalen, además, los instrumentos de molienda que por su tamaño y cantidad fueron considerados como artefactos con los que se dejaba equipado al sitio (Bayón y Flegenheimer 2004).

Entre los temas analizados en El Guanaco se destacan aquellos relacionados con la movilidad, los itinerarios y el constante retorno a las orillas de esta laguna.

Se ha considerado la movilidad de los grupos humanos que habitaron este entorno lagunar y las distancias que recorrieron a partir de las materias primas que trasladaron y el aprovechamiento que hicieron de las mismas, sugiriendo recorridos que incluyen la costa atlántica y los dos sistemas serranos (Figura 1) (Bayón y Flegenheimer 2004). A partir de las estrategias de abastecimiento de recursos líticos y el equipamiento del sitio con grandes cantidades de rocas trasladadas e instrumental de molienda se ha interpretado que los habitantes de estos lugares durante el Holoceno tardío contemplaban el retorno a este espacio durante sus recorridos (Bayón et al, 2006). Se considera que los cazadores recolectores en sus traslados fueron dejando los instrumentos de molienda en ciertos lugares debido a que su uso era estacional (Bayón y Flegenheimer 1998). 
Otra línea interpretativa se relaciona con los posibles recursos bióticos explotados. Los análisis de sustancias adheridas mediante CG-EM en combinación con los análisis de microfósiles de los instrumentos de molienda del Holoceno temprano y medio dan cuenta del procesamiento de vegetales, incluyendo hojas de Celtis tala que no habrían estado disponibles localmente (Babot et al. 2007). En tanto que, mediante estudios isotópicos sobre los restos humanos asignados al Holoceno tardío se ha inferido una dieta basada predominantemente en recursos continentales (Flegenheimer et al. 2002), desestimando la importancia del consumo de recursos de la costa atlántica, distante apenas $13 \mathrm{~km}$.

Finalmente, al considerar las prácticas mortuorias a comienzos del Holoceno tardío, resulta notable la presencia de entierros secundarios, en forma de conjunto o de arreglos de partes esqueletales aisladas (Mazzia et al. 2004). Todo entierro secundario implica la intencionalidad de modificar la disposición original de los restos humanos, ya sea para reacomodarlos en el mismo sitio o para trasladarlos a una nueva sepultura (Barley 2000; Borgognini Tarti y Pacciani 1993). En este caso, las partes esqueletales no solamente fueron reordenadas, sino que algunas de ellas no se encuentran presentes, implicando que posiblemente hayan sido trasladadas o separadas durante la preparación de los cuerpos. Esta particularidad remite a la posibilidad de que la misma práctica se haya llevado a cabos en diferentes lugares, dejando mediante el ritual mortuorio algo de cada difunto en diferentes locaciones. Se ha propuesto que El Guanaco representa un lugar elegido por la gente para enterrar a sus muertos mediante prácticas rituales particulares (Mazzia et al. 2004).

En suma, a partir de la integración de diferentes líneas de evidencias se propone que en las cercanías de la laguna El Lucero se hallan lugares del paisaje de los cazadores recolectores pampeanos a los que, desde hace aproximadamente 9000 años AP, volvían recurrentemente. Además, hace unos 2500 años AP un sector de este espacio humano fue resignificado como lugar mortuorio, sentido y funcionalidad que perduraron por un período de 700 años, aproximadamente.

\section{Otros sitios arqueológicos próximos a cuerpos lagunares}

El Guanaco no es un caso aislado en la región pampeana al sur de la depresión del Salado. Tanto en el área interserrana y en el sudoeste de la costa atlántica, como en ambos sistemas de sierras se repiten las ocupaciones y reocupaciones de los entornos lagunares (Tabla 1). Los ejemplos resultan escasos a comienzos del Holoceno pero luego comienzan a multiplicarse, principalmente durante el Holoceno tardío, evidenciando a la vez una mayor densidad de habitantes o visitas más frecuentes a las lagunas (Politis y Madrid, 2001). 


\begin{tabular}{|c|c|c|c|c|c|c|c|c|}
\hline Ref. & Sitio & $\begin{array}{l}\text { Cronología } \\
\text { Holoceno }\end{array}$ & \begin{tabular}{|l}
$\begin{array}{l}\text { Contexto } \\
\text { arqueológico }\end{array}$ \\
\end{tabular} & $\begin{array}{l}\text { Materias pri- } \\
\text { mas líticas }\end{array}$ & \begin{tabular}{|l} 
Recursos \\
lacustres
\end{tabular} & \begin{tabular}{|l} 
Prácticas \\
mortuorias
\end{tabular} & Caracterización & Ocupaciones \\
\hline 1 & La Colorada & Tardío & $\begin{array}{l}\text { AL, FAA e } \\
\text { introducida, } \\
\text { cerámica }\end{array}$ & $\begin{array}{l}\text { Más de } 100 \\
\text { km aprox. }\end{array}$ & $\begin{array}{l}\text { Sin evi- } \\
\text { dencias }\end{array}$ & sí & $\begin{array}{l}\text { Actividades } \\
\text { múltiples }\end{array}$ & Reocupación \\
\hline 2 & $\begin{array}{l}\text { Lag. de } \\
\text { Sotelo }\end{array}$ & Tardío & \begin{tabular}{|l|} 
AL, FAA, \\
cerámica, fauna \\
marina
\end{tabular} & $\begin{array}{l}\text { Más de } 150 \\
\text { km aprox. }\end{array}$ & $\begin{array}{l}\text { Sin evi- } \\
\text { dencias }\end{array}$ & no & $\begin{array}{l}\text { Campamento } \\
\text { base/actividades } \\
\text { múltiples }\end{array}$ & $\begin{array}{l}\text { Estacionalidad: } \\
\text { primavera }\end{array}$ \\
\hline 3 & Alfar & Medio & $\begin{array}{l}\mathrm{AL}, \mathrm{M}, \mathrm{FAA}, \\
\text { fauna marina }\end{array}$ & $\begin{array}{l}\text { Local- más de } \\
125 \mathrm{~km}\end{array}$ & $\begin{array}{l}\text { Sin evi- } \\
\text { dencias }\end{array}$ & no & $\begin{array}{l}\text { Actividades } \\
\text { múltiples }\end{array}$ & $\begin{array}{l}\text { Estacionalidad: } \\
\text { verano }\end{array}$ \\
\hline 4 & $\begin{array}{l}\text { Cueva La } \\
\text { Brava }\end{array}$ & Temprano & $\begin{array}{l}\text { AL, FAA, } \\
\text { fogones }\end{array}$ & $\begin{array}{l}\text { Local- más de } \\
60 \mathrm{~km}\end{array}$ & $\begin{array}{l}\text { Sin evi- } \\
\text { dencias }\end{array}$ & no & $\begin{array}{l}\text { Actividades } \\
\text { específicas }\end{array}$ & $\begin{array}{l}\text { Ocupación } \\
\text { efímera }\end{array}$ \\
\hline 5 & $\begin{array}{l}\text { Lag. La Bar- } \\
\text { rancosa }\end{array}$ & Tardío & $\begin{array}{l}\text { AL, FAA, } \\
\text { cerámica }\end{array}$ & Local & $\begin{array}{l}\text { Sin evi- } \\
\text { dencias }\end{array}$ & no & $\begin{array}{l}\text { Actividades espe- } \\
\text { cíficas en diferen- } \\
\text { tes sectores }\end{array}$ & - \\
\hline 6 & $\begin{array}{l}\text { El Guanaco } \\
1 \text { y } 2\end{array}$ & $\begin{array}{l}\text { Temprano } \\
\text { Medio } \\
\text { Tardío }\end{array}$ & $\begin{array}{l}\text { AL, M, FE, } \\
\text { FAA, cáscara } \\
\text { de huevo de } \\
\text { ńandú } \\
\text { AL, FAA, } \\
\text { objetos post } \\
\text { conquista }\end{array}$ & Más 100 km & $\begin{array}{l}\text { Sin evi- } \\
\text { dencias }\end{array}$ & $\begin{array}{l}1^{\circ}, \mathrm{PE} \\
1^{\circ} \mathrm{y} 2^{\circ} \mathrm{M}, \mathrm{PE}\end{array}$ & $\begin{array}{l}\text { Campamento } \\
\text { base/actividades } \\
\text { múltiples } \\
\text { Actividades } \\
\text { específicas }\end{array}$ & Reocupación \\
\hline 7 & $\begin{array}{l}\text { Arroyo } \\
\text { Seco } 2\end{array}$ & $\begin{array}{l}\text { Temprano/ } \\
\text { Medio } \\
\text { tardío }\end{array}$ & $\begin{array}{l}\text { AL, FE, FAA } \\
\text { AL, M, FAA }\end{array}$ & $50-170 \mathrm{~km}$ & $\begin{array}{l}\text { Sin evi- } \\
\text { dencias }\end{array}$ & $1_{\text {no }}^{1^{\circ}, 2^{\circ} \mathrm{S} \text { y } M}$ & \begin{tabular}{|l|} 
Campamento \\
base /actividades \\
múltiples y \\
específicas
\end{tabular} & Múltiples \\
\hline 8 & $\begin{array}{l}\text { Lag. Tres } \\
\text { Reyes }\end{array}$ & Tardío & $\begin{array}{l}\text { AL, FAA e } \\
\text { introducida, } \\
\text { cerámica }\end{array}$ & $\begin{array}{l}\text { Mayor a } 125 \\
\mathrm{~km}\end{array}$ & $\begin{array}{l}\text { Sin evi- } \\
\text { dencias }\end{array}$ & $1^{\circ} \mathrm{MyS}$ & \begin{tabular}{|l} 
Campamento \\
base /actividades \\
múltiples y \\
específicas \\
\end{tabular} & Reocupación \\
\hline 9 & Lag. La Larga & Tardío & $\begin{array}{l}\text { AL, FAA, } \\
\text { cerámica, } \\
\text { objetos post } \\
\text { conquista }\end{array}$ & $\begin{array}{l}\text { Al menos } \\
50 \mathrm{~km}\end{array}$ & $\begin{array}{l}\text { Sin evi- } \\
\text { dencias }\end{array}$ & $1 \circ \mathrm{S}$ y $\mathrm{M}$ & $\begin{array}{l}\text { Actividades } \\
\text { múltiples }\end{array}$ & - \\
\hline 10 & $\begin{array}{l}\text { Lag. del } \\
\text { Trompa }\end{array}$ & $\begin{array}{l}\text { Medio } \\
\text { Tardío }\end{array}$ & $\begin{array}{l}\text { AL, FAA, M } \\
\text { AL, M, pig- } \\
\text { mentos, FAA } \\
\text { e introducida, } \\
\text { cerámica }\end{array}$ & $80-200 \mathrm{~km}$ & $\begin{array}{l}\text { Sin evi- } \\
\text { dencias }\end{array}$ & no & $\begin{array}{l}\text { Campamento } \\
\text { base /actividades } \\
\text { múltiples y } \\
\text { específicas }\end{array}$ & $\begin{array}{l}\text { Estacionalidad: } \\
\text { invierno/ otońo } \\
\text { Reocupación }\end{array}$ \\
\hline 11 & $\begin{array}{l}\text { Fortín } \\
\text { Necochea }\end{array}$ & $\begin{array}{l}\text { Medio } \\
\text { Tardío }\end{array}$ & $\begin{array}{l}\text { AL, M, FAA } \\
\text { AL, FAA } \\
\text { e introdu- } \\
\text { cida, cerámica, } \\
\text { objetos post } \\
\text { conquista } \\
\end{array}$ & $80-200 \mathrm{~km}$ & $\begin{array}{l}\text { Sin evi- } \\
\text { dencias }\end{array}$ & no & $\begin{array}{l}\text { Campamento } \\
\text { base /actividades } \\
\text { múltiples }\end{array}$ & \begin{tabular}{|l} 
Retornos \\
planificados \\
Breves \\
Reocupación
\end{tabular} \\
\hline 12 & $\begin{array}{l}\text { Lag. La } \\
\text { Raquel } 2\end{array}$ & Tardío & $\begin{array}{l}\text { AL, FAA, } \\
\text { cerámica }\end{array}$ & $80-200 \mathrm{~km}$ & $\begin{array}{l}\text { Sin evi- } \\
\text { dencias }\end{array}$ & no & $\begin{array}{l}\text { Campamento } \\
\text { base /actividades } \\
\text { múltiples } \\
\end{array}$ & $\begin{array}{l}\text { Ocupación más } \\
\text { temprana en } \\
\text { discusión } \\
\end{array}$ \\
\hline 13 & $\begin{array}{l}\text { Monte Her- } \\
\text { moso } 1 \text { La } \\
\text { Olla } 1 \text { - } 2\end{array}$ & $\begin{array}{l}\text { Temprano } \\
\text { final / medio }\end{array}$ & \begin{tabular}{|l|} 
AL, M, \\
mamíferos ma- \\
rinos, artefactos \\
de madera \\
\end{tabular} & $\begin{array}{l}15-30 \mathrm{~km} \\
\text { aprox. }\end{array}$ & $\begin{array}{l}\text { Sin evi- } \\
\text { dencias }\end{array}$ & PE & $\begin{array}{l}\text { Actividades espe- } \\
\text { cíficas próximas } \\
\text { a campamento } \\
\text { base }\end{array}$ & \\
\hline 14 & $\begin{array}{l}\text { Lag. Los } \\
\text { Chilenos }\end{array}$ & Tardío & \begin{tabular}{|l|} 
cerámica, \\
cuentas de \\
valvas, molusco \\
marino, AL, \\
FAA \\
\end{tabular} & $30 \mathrm{~km}$ aprox. & $\begin{array}{l}\text { Sin evi- } \\
\text { dencias }\end{array}$ & $\begin{array}{l}2^{\circ} \mathrm{M}, 1^{\circ} \\
\mathrm{PE}\end{array}$ & $\begin{array}{l}\text { S1: Área de } \\
\text { inhumaciones } \\
\text { S2: Actividades } \\
\text { múltiples }\end{array}$ & Reocupación \\
\hline 15 & Lag. de Púan & Tardío & $\begin{array}{l}\text { Escondrijo } \\
\text { materias } 1^{\circ} \\
\text { líticas, FAA }\end{array}$ & $40 \mathrm{~km}$ aprox. & $\begin{array}{l}\text { Sin evi- } \\
\text { dencias }\end{array}$ & $2^{\circ}$ & $\begin{array}{l}\text { Actividades } \\
\text { específicas }\end{array}$ & \\
\hline 16 & $\begin{array}{l}\text { Las Brus- } \\
\text { quillas }\end{array}$ & Tardío & $\begin{array}{l}\text { AL, FAA, } \\
\text { cerámica }\end{array}$ & $60-80 \mathrm{~km}$ & $\begin{array}{l}\text { Sin evi- } \\
\text { dencias }\end{array}$ & no & $\begin{array}{l}\text { Campamento } \\
\text { base/ actividades } \\
\text { múltiples }\end{array}$ & $\begin{array}{l}\text { Breves/ no } \\
\text { redundantes } \\
\text { Reocupación }\end{array}$ \\
\hline
\end{tabular}

Tabla 1: Descripción de sitios arqueológicos en cercanias de lagunas. Referencias: $A L-$ artefactos líticos; FAA-fauna autóctona actual; M-molienda; FE-fauna extinta; PE-

$$
\text { partes esqueletales; } S \text {-simple; } M \text { - múltiple }
$$

Table 1: Description of archaeological sites next to lagoons. References: $A L$ - lithic artifacts; FAA-native fauna; $M$-grinding artifacts; FE-extinct fauna; PE- skeletal elements; S-simple; $M$ - multiple. 
A comienzos del Holoceno se han registrado ocupaciones humanas en los sitios Arroyo Seco 2, Cueva la Brava y en El Guanaco 1 y 2. Hacia el final del Holoceno temprano se suman los registros en Monte Hermoso 1 y La Olla 1 y 2. Arroyo Seco 2 (Politis 2008) se localiza en una lomada en las adyacencias de una laguna temporaria y del curso del arroyo Seco (Figura 1:7); con fechados de más de 12.000 años AP, marca la llegada de los primeros habitantes a la región. El sitio es considerado un campamento base que fue ocupado recurrentemente, en donde se llevaron a cabo diferentes actividades domésticas. Durante las ocupaciones tempranas se registra además la definición de este espacio como un lugar mortuorio. Los entierros secundarios evidencian la ausencia de ciertas partes esqueletales y hacen referencia a la manipulación de los cuerpos después de la muerte (Barrientos 1997, Politis 2008, Scabuzzo y Politis 2007).

Cueva La Brava se encuentra en el extremo oriental del sistema serrano de Tandilia, próximo a la cima del Cerro Valdés y frente a una laguna de aguas permanentes (Figura 1:4). Las evidencias humanas en este espacio fueron interpretadas como el producto de una ocupación efímera asociada con las últimas etapas de manufactura lítica enmarcadas por la caza de animales (Mazzanti 1999). Se trata de un emplazamiento diferente a los anteriores por hallarse en un abrigo rocoso.

Monte Hermoso 1, La Olla 1 y 2 (Bayón et al. 2011) son áreas arqueológicas relacionadas entre sí, interpretadas como parte de un mismo sistema de asentamiento en los bordes de una antigua laguna salobre, ubicada entre los médanos en la costa sudoeste de la región pampeana (Figura 1:13). La Olla 1 y 2 podrían representar espacios en los que sellevó a cabo el procesamiento secundario de lobos marinos y la explotación de recursos vegetales (Bayón et al. 2011, Politis 2008). En Monte Hermoso 1 se han registrado centenares de pisadas humanas preservadas en depósitos limoarenosos. La mayor parte de las huellas corresponden a niños, jóvenes y posiblemente a mujeres por lo que se propuso que el lugar de acampe debería estar establecido muy cerca de donde se identificaron las huellas (Bayón et al. 2011).

Durante el Holoceno medio continúan las ocupaciones humanas en los sitios Arroyo Seco 2 y El Guanaco 1 y 2, al mismo tiempo que nuevas orillas son visitadas y nuevas lomadas comienzan a ser elegidas como lugares en la llanura interserrana: los sitios Fortín Necochea y Laguna del Trompa (Figura 1:11 y 10) y en la costa atlántica: Alfar (Figura 1:3). Las ocupaciones humanas en Arroyo Seco 2 (Politis 2008) y Fortín Necochea (Crivelli Montero et al. 1987/88 a y b) tienen características similares en cuanto a su ubicación en sectores elevados, cercanos a bordes de lagunas, en la zona central 
de esta llanura, distantes de los sistemas serranos y de la costa atlántica. En ambos sitios se ha inferido la realización de diversas actividades domésticas relacionadas con los campamentos base de los grupos pampeanos (Politis 2008, Crivelli Montero et al. 1987/88, a y b). En Fortín Necochea se han hallado agrupamientos de implementos de molienda y depósitos de lascas que evidencian la intención de retornar al lugar (Crivelli Montero et al. 1987/88, a y b). En Arroyo Seco 2 se registran nuevamente entierros humanos que dan cuenta de la persistencia de este espacio como un lugar mortuorio durante, al menos, 3000 años (Scabuzzo y Politis 2007).

El sitio Laguna del Trompa se encuentra a orillas de un espejo de agua permanente, que representa una de las lagunas de mayor tamaño del área. A partir del contexto arqueológico se ha inferido la recurrencia de ocupaciones y el desarrollo de actividades diversas. Incluso se han definido áreas en donde se realizaron tareas específicas, como por ejemplo un episodio localizado de reparación de puntas de proyectil (Crivelli Montero 1991, Eugenio 1991, Silveira 1991).

Por último, Alfar está ubicado sobre el borde de un antiguo cuerpo de agua en la zona de médanos, próximo al curso inferior del arroyo Corrientes y a $650 \mathrm{~m}$ de la actual línea de costa. Presenta evidencias de una importante explotación de lobos marinos, que habrían estado disponibles en las colonias próximas durante el verano, y de un aprovechamiento de nódulos costeros como materia prima lítica (Bonomo y León 2010).

Las evidencias de ocupación humana en la región pampeana durante el Holoceno tardío, son mucho más abundantes y los sitios de actividades múltiples a orillas de lagunas muestran una mayor densidad de materiales sugiriendo cierta redundancia en las ocupaciones o estadías más prolongadas (Politis y Madrid 2001). Además de los lugares que persisten a lo largo del tiempo como El Guanaco 1, Arroyo Seco 2, Fortín Necochea y Laguna del Trompa, surgen nuevos espacios humanos en cercanías de lagunas con una distribución amplia.

Al noroeste de Fortín Necochea y Laguna del Trompa se encuentra el sitio arqueológico Laguna La Raquel 2, sobre la parte más alta de una lomada en la orilla este de una laguna de aguas permanentes (Figura 1:12). El sitio fue interpretado como un campamento base en el que se desarrollaron actividades diversas (Silveira et al. 1997).

La localidad arqueológica Laguna de Púan (Figura 1:15) se encuentra al sudoeste del sistema serrano de Ventania; incluye cinco sitios, cuatro 
ubicados en una isla de $6 \mathrm{~km}^{2}$ hacia el centro de la laguna y otro en su costa norte. Allí se ha registrado el hallazgo de un reservorio de riolita, materia prima de distribución muy restringida, que aflora a más de $40 \mathrm{~km}$ de distancia. Esta roca fue encontrada formando un agrupamiento de nódulos, núcleos y artefactos acomodados (Oliva et al. 1991).

Hacia el noreste de Laguna de Púan se encuentra la localidad Laguna Los Chilenos, en las proximidades de un cuerpo de agua permanente ubicado $15 \mathrm{~km}$ hacia el sudoeste de dos pequeñas sierras de Ventania (Figura 1:14). Sobre la costa sur de esta laguna se hallaron entierros humanos que presentaban coloración de los cuerpos con pigmentos rojos y amarillos. Este sector del entorno lagunar fue interpretado como un área específica para inhumaciones. A $700 \mathrm{~m}$ en dirección oeste se registró un espacio ocupado intensamente. Estos dos lugares de cazadores recolectores tardíos habrían sido contemporáneos durante los últimos 1000 años AP (Barrientos et al. 1997, Barrientos et al. 2002).

La localidad Laguna La Larga (Figura 1:9) está definida por tres sectores en el margen este, en la barranca y en la playa de una laguna de 400 ha de superficie, en la llanura interserrana. Los sitios 1, 2 y 3 presentan diversas evidencias entre las que se incluyen entierros humanos (Barrientos 1997, Pérez 2002).

Otro lugar mortuorio del Holoceno tardío se encuentra representado por el sitio Laguna Tres Reyes 1, ubicado sobre la orilla norte de la laguna (Figura 1:8). Ese lugar fue elegido para inhumar los cuerpos de personas en disposición primaria. Se trata de un entierro múltiple compuesto por nueve individuos que interceptó a un entierro preexistente. Por ello, se infirió la persistencia en la elección de este espacio para el desarrollo de prácticas mortuorias (Madrid et al. 1991, Madrid y Salemme 1991).

El sitio Laguna La Barrancosa se encuentra en la llanura serrana ubicada en el sector noroeste de Tandilia, muy próximo al curso superior del arroyo Tapalqué (Figura 1:5). Este sitio arqueológico se localiza en el sector norte de la laguna, sobre una barranca. En las proximidades de esta laguna se habría producido el procesamiento secundario de guanacos y se habría realizado la formatización, reactivación y reciclado de artefactos líticos (Gómez y Messineo 2008, Pal 2008).

Cerca de la costa atlántica, al noreste del extremo oriental de Tandila se encuentra el sitio Laguna de Sotelo (Figura 1:2). Se trata de un espacio en el que se llevaron a cabo diversas actividades relacionadas con la talla de instrumentos líticos, la manufactura cerámica y el procesamiento y consumo 
de venado de las pampas, coipo, huevos de ñandú, corvinas y un cetáceo. Esto último sugiere un aprovechamiento ocasional de los recursos marinos próximos (Eugenio y Pardiñas 1991).

En el mismo sector centro-sur del área interserrana que El Guanaco, se encuentra La localidad arqueológica Las Brusquillas (Figura1:16). Allí se identificó la existencia de un cuerpo de agua somero durante el Holoceno tardío. En el sitio 1 las ocupaciones humanas habrían sido de carácter residencial, aunque breves o no redundantes, persistiendo hasta momentos post-conquista. En tanto en el sitio 2 se identificaron actividades de caza y características domésticas (Massigoge 2011, 2012).

Finalmente, el sitio La Colorada se encuentra sobre el borde noreste de la laguna homónima (Figura 1:1). Allí se han recuperado restos óseos humanos y evidencias del desarrollo de actividades diversas (Aldazábal et al. 2004).

\section{Discusión}

A lo largo del Holoceno diferentes entornos lagunares pampeanos, ubicados al sur del río Salado, han sido visitados y habitados, en muchos casos de forma recurrente. La reutilización de estos espacios fue considerada, en líneas generales, como el resultado de eventos separados pero que pueden haber tenido cierta relación entre sí (Martínez 1999). Al respecto, las interpretaciones formuladas varían tanto como las características de los sitios descriptos. Desde la perspectiva generada a partir de ciertos ejemplos se ha propuesto que los sitios en ambientes fluviales y/o lagunares pueden considerarse sitios de actividades específicas básicamente relacionados a la caza y al procesamiento inicial de presas (Martínez 1999). En tanto que, al considerar otro tipos de sitios, se ha planteado que desde el Holoceno medio se hace evidente la utilización recurrente de las márgenes de cuerpos lagunares para la localización de campamentos residenciales (Politis y Madrid 2001, Aldazábal et al. 2004). Una propuesta similar expresa que en la región pampeana los cuerpos de agua debieron actuar en el pasado como importantes concentradores de poblaciones humanas a través del tiempo (Barrientos et al. 1997). En esta línea, se entiende que las decisiones de los grupos humanos acerca del uso de estos espacios, como escenario para la realización de determinadas actividades, pueden ser consideradas como soluciones apropiadas u óptimas frente a los problemas creados por la distribución de recursos críticos como combustible, agua, presas y materias primas, situación que influiría en su reutilización (Barrientos et al. 1997). 
Sin embargo, en contraposición a esta última propuesta, en la mayoría de los casos descriptos anteriormente, las lagunas están situadas a distancias considerables de las principales fuentes de materias primas líticas utilizadas. A esto debe sumarse que en la pampa bonaerense los recursos líticos se encuentran muy localizados en el espacio. Por tal motivo, el emplazamiento en las proximidades de las lagunas requirió una planificación de la obtención y el traslado de importantes cantidades de rocas que fueron dejadas en los lugares a los que los grupos pensaban volver. En este sentido, es notoria la presencia de reservorios de materias primas líticas en los sitios Laguna de Puán y Fortín Necochea, como también la preparación de los núcleos para su máximo aprovechamiento en la localidad El Guanaco. También resulta destacable la presencia de gran cantidad de implementos de molienda en lugares como Arroyo Seco 2, El Guanaco, Fortín Necochea y La Olla. Además, en ninguno de estos sitios pudieron identificarse evidencias de un aprovechamiento intensivo de los recursos lacustres, como peces o aves.

La presencia de entierros humanos en disposición secundaria implica decisiones sociales de retorno a estos espacios que van más allá del aprovechamiento de los recursos que estos puedan ofrecer. Estas decisiones involucran la elección de un lugar para enterrar a los muertos y para el desarrollo de prácticas mortuorias particulares. Más aún, la separación y el traslado de partes esqueletales permite pensar en las relaciones materiales entre diferentes lugares que los grupos humanos habrían establecido mediante los rituales mortuorios, al enterrar partes de sus difuntos en diferentes puntos del espacio recorrido. En sitios como El Guanaco, Laguna Los Chilenos 1 y Arroyo Seco 2 existió además una persistencia de esta elección a través del tiempo.

Por supuesto, también existen evidencias de sitios con ocupaciones efímeras como Cueva La Brava o más acotadas en el tiempo como parecieran ser Monte Hermoso 1 y La Olla 1 y 2, Laguna La Barrancosa, Alfar, Las Brusquillas o Laguna de Sotelo.

Puede proponerse entonces, que no existe un único parámetro que resulte útil para definir las ocupaciones humanas en las lagunas. Claramente no es el ambiente lagunar en sí mismo el que determina cómo van a aprovechar ese espacio las personas que se acerquen a sus orillas. En cambio, es en el continuo movimiento, en los recorridos que realizaban durante las distintas estaciones los cazadores recolectores, que estos lugares cobraron sentido.

Indudablemente, la presencia de agua debe haber tenido un rol fundamental en todos los sentidos otorgados a estos lugares. Uno de los 
aspectos más obvios sobre el agua es que representa un elemento esencial para la supervivencia de todo ser vivo. En tal sentido, las aguas de las lagunas, como recurso, deben haber atraído no sólo a los grupos humanos sino también a una gran diversidad de animales. Además, su presencia permite el crecimiento y desarrollo de una importante variedad de plantas. Es cierto que el agua puede ser considerada un recurso natural y una necesidad vital, pero también puede ser concebida socialmente como un bien para ser poseído o, incluso, un objeto sagrado (Pińeyro 2006). El rol del agua para los seres humanos cambia al tiempo que toma la forma de una bebida, de un elemento para la higiene y la salud, para la supervivencia y la reproducción del mundo natural o para el desarrollo de diferentes rituales que marcan la vida social. Por otra parte, la superficie del agua puede comportarse como una fuente luminosa al reflejar la luz que incide sobre ella. De igual forma, el agua ha actuado durante la mayor parte de la historia humana como un espejo que permitió a las personas tener una imagen de sí mismas (Strang 2005).

Como sustancia compartida en un territorio, el agua puede unir o dividir a diferentes grupos sociales, física y topográficamente: en forma de ríos, arroyos, lagos y lagunas crea fronteras, divide u ordena y organiza el espacio (Richards 1996, Strang 2005). El movimiento o fluir del agua desde sus nacientes en sierras y montañas proveen un símbolo del movimiento, de los recorridos, de los caminos (Richards 1996). En contraposición las aguas quietas de lagos y lagunas tienen más que ver con la pausa, y la pausa con los lugares. Las lagunas pampeanas representan lugares que formaban parte de la vida de los grupos de cazadores recolectores, quienes, en ciertos casos, volvieron una y otra vez a sus orillas. El regreso puede haber estado motivado por la disponibilidad de agua, por la abundancia de recursos vegetales y animales que se podían encontrar en sus alrededores, por la presencia de artefactos que equipaban los sitios, por los reservorios de materias primas líticas o por las tumbas de quienes los antecedieron. Aunque quizás todos estos motivos sean las consecuencias, y no las causas, de la elección de un determinado lugar por su historia y por sus significados como parte de su paisaje.

\section{Conclusiones}

Espacio y lugar son componentes básicos del mundo vivido por los seres humanos; ambos se definen por el movimiento y las experiencias subjetivas (Bachelard, 1965, Tuan, 2008 [1977]). El entramado de lugares de los cazadores recolectores toma su forma a partir de los caminos que los unen y las distancias que los separan. Las distancias recorridas entre un 
punto y otro remiten a grados de accesibilidad, de necesidades e intereses. Cada uno de estos puntos en el espacio da cuenta de las elecciones tomadas durante el trayecto. Al conectar diferentes lugares, los caminos materializan al movimiento de los cuerpos a través del espacio (Potter 2004, Tilley 1994). Por ello se considera que el espacio permite el movimiento, en tanto que los lugares representan la pausa (Tuan 2008 [1977]). El paisaje de los cazadores recolectores se basa en un entramado de pausas y movimientos, de lugares y caminos.

En este marco, las fuentes de agua, recurso vital y cargado de significados, siempre son incluidas en los trayectos recorridos. Como punto de partida, de llegada o como un alto en el camino, las fuentes de agua resultan una referencia ineludible. En tal sentido, los entornos del agua quieta en las lagunas son entendidos como una pausa en el espacio de los cazadores recolectores. Las orillas lagunares se transforman, según el contexto, en vivienda, lugar mortuorio o una breve pausa para el descanso, para obtener alimentos o para manufacturar artefactos: un lugar en el paisaje de los cazadores recolectores.

Agradecimientos: Quiero agradecer a N. Flegenheimer, I. González, M. Bonomo y C. Bayón por los comentarios y sugerencias realizados a una versión previa del manuscrito. Además, este trabajo se nutrió de las experiencias compartidas en los trabajos de campo con C. Scabuzzo, R. Vecchi, R. Frontini, A. Pupio, C. Weitzel, M. Colombo y C. Pérez Levalle. Un agradecimiento especial es para los propietarios y empleados de la Estancia El Guanaco y para el Sr. V. Bustillo y el personal de Proagro. Esta investigación fue realizada en el marco de los proyectos El uso del paisaje $y$ los recursos minerales de los grupos cazadores-recolectores en las Sierras de Tandilia, PIP 112-200801-02979. 2009/2011, CONICET, dirigido por N. Flegenheimer, y Dinámica social, escenarios y materialidad entre los cazadores recolectores pampeanos, PICT 2010-1517. 2011-2013, ANPCyT, dirigido por I. González.

\section{Notas}

Los sitios Laguna Arroyo Venado (Oliva et al., 2012) y Laguna Salalé (Oliva et al., 2004) no fueron incluidos en la discusión por no contar hasta el momento con información cronológica publicada.

Los materiales líticos de esta colección han sido atribuidos al Holoceno tardío (Bayón y Flegenheimer, 1998; Vecchi et al., 2007), sin embargo no puede descartarse la existencia de objetos de antigüedades diferentes en el mismo conjunto (Favier-Dubois y Bonomo, 2008). 


\section{BiBLIOGRAFÍA}

Aldazábal, V.; N. Weiler y E. Eugenio. 2004. "Una perspectiva geoarqueológica para comprender la ocupación humana en la costa central de la provincia de Buenos Aires". Intersecciones en Antropología 5: 29-38.

Augé, M. 1998. Los No Lugares. Espacios del Anonimato. Una Antropología de la Sobremodernidad. Editorial Gedisa, Barcelona.

Babot, M.; N. Mazzia y C. Bayón. 2007. "Procesamiento de recursos en la región pampeana bonaerense: aportes del instrumental de molienda de las localidades arqueológicas El Guanaco y Cerro La China”. En Arqueología en las Pampas, editado por C. Bayón, N. Flegenheimer, M. I. González, M. Frere y A. Pupio, pp: 635-660. SAA-UNS, Bahía Blanca.

Bachelard, G. 1965. La poética del espacio. Fondo de Cultura Económica, México.

Barley, N. 2000. Bailando Sobre la Tumba. Anagrama, Barcelona.

Barrientos, G. 1997. Nutrición y Dieta de las Poblaciones Aborígenes Prehispánicas del Sudeste de la Región Pampeana. Tesis para optar al grado de doctor en Ciencias Naturales, Facultad de Ciencias Naturales y Museo, UNLP, La Plata.

Barrientos, G.; M. Leipus y F. Oliva. 1997. "Investigaciones arqueológicas en la laguna Los Chilenos (provincia de Buenos Aires)". En Arqueología de la Región Pampeana en la década de los '90, editado por M. Berón y G. Politis, pp: 115-125. Museo Municipal de Historia Natural de San Rafael e INCUAPA, San Rafael.

Barrientos, G.; F. Oliva y M. del Papa. 2002. "Historia pre y postdepositacional del entierro secundario del sitio Laguna Los Chilenos 1 (provincia de Buenos Aires)". Relaciones de la Sociedad Argentina de Antropología XXVII: 303-325.

Bayón, C. y N. Flegenheimer. 1998. "Un caso de aplicación: procedencia de rocas en sitio El Guanaco". Trabajo presentado en el I Congreso Nacional de Arqueología Pampeana. Santa Fe, Argentina.

__ 2004. "Cambio de planes a través del tiempo para el traslado de roca en la pampa bonaerense”. Estudios Atacameños 28: 59-70.

Bayón, C.; N. Flegenheimer, M. Zárate y C. Deschamps. 2004. ““...Y vendrán los arqueólogos en busca de un hueso"... Sitio El Guanaco, partido de San Cayetano". En Aproximaciones Arqueológicas Pampeanas. Teorías, Métodos y Casos de Aplicación Contemporáneos, editado por G. Martínez, M. Gutiérrez, R. Curtoni, M. Berón y P. Madrid, pp: 247-258. Facultad de Ciencias Sociales, UNCPBA, Olavarría. 
Bayón, C.; N. Flegenheimer y A. Pupio. 2006. "Planes sociales en el abastecimiento y traslado de roca en la pampa bonaerense en el Holoceno temprano y tardío". Relaciones de la Sociedad Argentina de Antropología XXXI: $19-45$.

Bayón, C.; T. Manera, G. Politis y S. Aramayo. 2011. "Following the tracks of the first South Americans". Evolution: Education and Outreach, 4: 205217.

Bender, B. 2002. "Time and Landscape". Current Anthropology, 43: 103-137.

Bonomo, M. y C. León. 2010. "Un contexto arqueológico en posición estratigráfica en los médanos litorales. El sitio Alfar (Pdo. Gral. Pueyrredón, Pcia. Bs. As.)”. En Mamül Mapu: Pasado y Presente desde la Arqueología Pampeana, editado por M. Berón, L. Luna, M. Bonomo, C. Montalvo, C. Aranda y M. Carrera Aizpitarte, pp: 241-252. Ediciones del Espinillo, Buenos Aires.

Borgognini Tarti, S. y E. Pacciani. 1993. I Resti Umani Nello Scavo Archeologico. Metodiche di Recupero e Studio. Bulzoni, Roma.

Carden, N. 2008. Imágenes a Través del Tiempo. Arte rupestre y Construcción Social del Paisaje en la Meseta Central de Santa Cruz. SAA, Buenos Aires.

Criado Boado, F. 1999. "Del terreno al espacio: planteamientos y perspectivas para la arqueología del paisaje”. CAPA 6: 1-63.

Crivelli Montero, E. 1991. "Laguna la Trompa (estancia La Herminia), Laprida, pcia. de Bs. As. Excavaciones 1989-1991. Artefactos y estructuras”. Boletín del centro 3: 18-29.

Crivelli Montero, E.; E. Eugenio y M. Silveira. 1987/88a. "El sitio Fortín Necochea (provincia de Buenos Aires). El material de superficie”. Paleoetnológica IV: 7-37.

Crivelli Montero, E.; M. Silveira, E. Eugenio, P. Escola, M. Fernández y N. Franco. 1987/88b. "El sitio Fortín Necochea (partido de Gral. Lamadrid, provincia de Buenos Aires). Estado actual de los trabajos”. Paleoetnológica IV: 39-53.

Curtoni, R. 2007. Arqueología y Paisaje en el Área Centro-Este de La Pampa. Tesis para optar al grado de doctor en Ciencias Naturales, Facultad de Ciencias Naturales y Museo, UNLP, La Plata.

Dangavs, N. 2005. "Los ambientes acuáticos de la provincia de Buenos Aires". En Geología y Recursos Minerales de la Provincia de Buenos Aires. Relatorio del XVI Congreso Geológico Argentino, editado por R. E. de Barrio, R. O. Etcheverry, M. F. Caballé y E. Llambías, pp: 219-236. La Plata, Argentina. 
Diez Martín, F. 2007. "La arqueología del paisaje en la investigación paleolítica". Arqueoweb. Revista sobre arqueología en Internet 9 (1).

Eugenio, E. 1991. "Asentamientos arqueológicos en la laguna del Trompa (estancia La Herminia), Laprida, pcia de Bs. As.” Boletín del centro 3: 30-42.

Eugenio, E. y Pardiñas, U. 1991. "Zooarqueología del sitio Laguna de Sotelo. Partido de Mar Chiquita, pcia. de Bs. As.”. Boletín del centro 3: 43-52.

Favier-Dubois, C. y M. Bonomo. 2008. "Geoarqueología en la localidad Nutria Mansa (Pdos. de Gral. Alvarado y Lobería, provincia de Buenos Aires)”. Comechingonia 11: 9-28.

Flegenheimer, N.; R. A. Guichón y C. Scabuzzo. 2002. "Restos óseos humanos en el sitio El Guanaco, partido de San Cayetano". En Del Mar a los Salitrales. Diez Mil Años de Historia Pampeana en el Umbral del Tercer Milenio, editado por D. Mazzanti, M. Berón y F. Oliva, pp: 121-126. UNMdPSAA, Buenos Aires.

Flegenheimer, N.; C. Bayón, C. Scabuzzo, N. Mazzia, R. Vecchi, C. Weitzel, R. Frontini y M. Colombo. 2010. "Early Holocene human skeletal remains from the Argentinean Pampas". Current Research in the Pleistocene $27: 10-12$.

Frenguelli, J. 1956. "Rasgos generales de la hidrografía de la Provincia de Buenos Aires”. LEMIT 62 (2): 1-19.

Frontini, R. 2010. "Las arqueofaunas en la cocina. Actividades culinarias en el sitio 2 de la localidad arqueológica El Guanaco". Arqueología 16: 191-208.

- 2012. El Aprovechamiento de Animales en Valles Fluviales y Lagunas del Sur Bonaerense durante el Holoceno. Tesis para optar al grado de doctor en Arqueología. Facultad de Filosofía y Letras, UBA, Buenos Aires.

Frontini, R. y M. Picasso. 2010. "Aprovechamiento de Rhea americana en la Localidad Arqueológica El Guanaco". En Zooarqueología a Principios del Siglo XXI: Aportes Teóricos, Metodológicos y Casos de Estudio, editado por M. Gutiérrez, M. De Negris, P. Fernández, M. Giardina, A. Gil, A. Izeta, G. Neme y H. Yacobaccio, pp: 563-574. Ediciones del Espinillo, Buenos Aires.

Gómez, G. y P. Messineo. 2008. “Análisis tafonómico de micromamíferos y mesomamíferos del sitio Laguna La Barrancosa 1 (Partido de Benito Juárez, provincia de Buenos Aires)". Intersecciones en Antropología 9: 71-91.

González, M. I. 2005. Arqueología de Alfareros, Cazadores y Pescadores Pampeanos. SAA, Buenos Aires. 
González, M. I.; M. M. Frère y P. Escosteguy. 2006. "El sitio San Ramón 7. Curso inferior del río Salado, provincia de Buenos Aires". Relaciones de la Sociedad Argentina de Antropología XXXI: 187-199.

Ingold, T. 1993. "The temporality of the Landscape". World Archaeology 25 (2): 152-174.

- 2000. The Perception of the Environment. Essays on Livelihood, Dwelling and Skill. Routledge, Londres-Nueva York.

Low, S. M. 2003. "Embodied Space(s). Anthropological Theories of body, Space and Culture". Space and Culture 6 (1): 9-18.

Madrid, P. y M. Salemme. 1991. "La ocupación tardía del sitio 1 de la Laguna Tres Reyes. Partido de Adolfo González Chávez, pcia. de Bs. As”. Boletín del centro 3:165-179.

Madrid, P.; G. Politis, M. Leipus y C. Landini. 1991. "Estado actual de las investigaciones en el sitio 1 de la Laguna Tres Reyes: análisis lítico tecnomorfológico y procesos de formación del sitio". Boletín del centro 2:112122.

Martínez, G. 1999. Tecnología, Subsistencia y Asentamiento en el Curso Medio del Rio Quequén Grande: Un Enfoque Arqueológico. Tesis para optar al grado de doctor en Ciencias Naturales, Facultad de Ciencias Naturales y Museo, UNLP, La Plata.

Massigoge, A. 2011. "Nuevas evidencias arqueológicas del Holoceno Tardío en el área Interserrana: el sitio Las Brusquillas 2 (partido de San Cayetano, provincia de Buenos Aires, Argentina)". Cazadores-Recolectores del Cono Sur. Revista de Arqueología 5: 180-195.

- - 2012. "Las Brusquillas 1 (partido de San Cayetano, provincia de Buenos Aires): un nuevo sitio del Holoceno tardío del área Interserrana de la región pampeana”. Intersecciones en Antropología 13: 377-392.

Mazzanti, D. L. 1999. "Ocupaciones humanas tempranas en Sierra La Vigilancia y Laguna La Brava, Tandilia Oriental (provincia de Bs. As.)”. Actas del XII Congreso Nacional de Arqueología Argentina, Vol III, pp: 149-155. La Plata, Argentina.

Mazzanti, D. L. y F. Valverde. 2003. "Representaciones rupestres de cazadoresrecolectores en las sierras de Tandilia oriental: una aproximación a la Arqueología del Paisaje”. Actas del XIII Congreso Nacional de Arqueología Argentina, Tomo III, pp: 311-316. Córdoba, Argentina.

Mazzia, N. 2010-2011. Lugares y Paisajes de Cazadores Recolectores en la Pampa Bonaerense: Cambios y Continuidades Durante el Pleistoceno Final - Holo- 
ceno. Tesis para optar al grado de doctor en Ciencias Naturales, Facultad de Ciencias Naturales y Museo, UNLP, La Plata.

__ 2013. "Lugares y paisajes de cazadores recolectores pampeanos: una propuesta para su estudio". Revista del Museo de La Plata, Sección Antropología 13: 233-253.

Mazzia, N.; C. Scabuzzo y R. Guichón. 2004. "Sobre cráneos, pelvis y otros huesos. Entierros humanos en el sitio El Guanaco". En Aproximaciones Arqueológicas Pampeanas. Teorías, Métodos y Casos de Aplicación Contemporáneos, editado por G. Martínez, M. Gutiérrez, R. Curtoni, M. Berón y P. Madrid, pp. 293-304. Facultad de Ciencias Sociales, UNCPBA, Olavarría.

Oliva, F.; J. Moirano y M. Saghessi. 1991. "Estado de las investigaciones arqueológicas en el sitio Laguna de Puán 1”. Boletín del Centro 2:127-138.

Oliva, F.; J. Ávila, M. Gallego y M. Algrain. 2004. "Investigaciones arqueológicas en la laguna Salalé (Partido Florentino Ameghino, Provincia de Buenos Aires). Una aproximación al uso de los recursos líticos". En La Región Pampeana, Su Pasado Arqueológico, editado por C. Gradin y F. Oliva, pp: 377-387. Laborde Editor, Rosario.

Oliva, F.; L. Iannelli y B. Pereyra. 2012. "Presentación de las tareas de rescate en Laguna Arroyo Venado, provincia de Buenos Aires”. Anuario de Arqueología 4: $147-159$.

Pal, N. 2008. "Aportes al estudio de la integridad del sitio Laguna La Barrancosa 1 (partido de Benito Juárez, provincia de Buenos Aires): análisis textural y distribucional de los microdesechos líticos”. Intersecciones en Antropología 9: 59-75.

Pérez, S. I. 2002. "Variabilidad temporal en la morfología craneofacial en muestras de restos humanos del sudeste de la región pampeana: implicaciones para la discusión del poblamiento regional durante el holoceno". En Del Mar a los Salitrales. Diez Mil Años de Historia Pampeana en el Umbral del Tercer Milenio, editado por D. Mazzanti, M. Berón y F. Oliva, pp: 155170. Universidad Nacional de Mar del Plata-SAA, Buenos Aires.

Pińeyro, N. 2006. "Agua y Semiótica". Revista On-Line de la Universidad Bolivariana Vol. 5 (14).

Politis, G. 2008. "The Pampas and Campos of South America”. En Handbook of South American Archaeology, editado por H. Silverman y W. Isbell, pp: 235-260. Springer, Nueva York.

Politis, G. y P. Madrid. 2001. "Arqueología pampeana: estado actual y perspec- 
tivas". En Historia Argentina Prehispánica, editado por E. Berberián y A. Nielsen, Tomo II, pp: 737-813. Editorial Brujas, Córdoba.

Potter, J. 2004. "The creation of person, the creation of place: Hunting landscapes in the American Southwest". American Antiquity 69 (2): 322- 338.

Richards, C. 1996. "Henges and water. Towards an elemental understanding of monumentality and lanscape in late Neolithic Britain”. Journal of Material Culture 1: 313-336.

Scabuzzo C. y G. Politis. 2007. "Early Holocene secondary burials in the Pampas of Argentina". Current Research of the Pleistocene 23: 64-66.

Silveira, M.J. 1991. "Análisis de los restos óseos de las excavaciones de los sectores H y J del sitio Laguna del Tronpa. Estancia La Herminia, Laprida, Bs. As”. Boletín del centro 3: 105-115.

Silveira, M.; A. Menegaz y N. Weiler. 1997. "Sitio 2 La Raquel (Partido de General La Madrid, provincia de Buenos Aires). Fauna extinta y asociación cultural: discusión". En Arqueología de la Región Pampeana en la década de los '90, editado por M. Berón y G. Politis, pp: 239-250. Museo Municipal de Historia Natural de San Rafael e INCUAPA, San Rafael.

Strang, V. 2005. "Common senses. Water, Sensory Experience and the Generation of Meaning”. Journal of Material Culture 10(1): 92-120.

Taçon, P. 1994. "Socialising landscapes: the long-term implications of signs, symbols and marks on the land”. Archaeology in Oceania 29 (3): 117- 129.

Thomas, J. 2001. "Archaeologies of place and landscape". En Archaeological Theory Today, editado por I. Hodder, pp. 165-186. Polity Press, Cambridge.

Tilley, C. 1994. A Phenomenology of Landscape, Places, Paths and Monuments. Berg, Oxford/Providence.

Troncoso, A. 1999. "De las sociedades en el espacio a los espacios en las sociedades: sobre Arqueología y Paisaje". Boletin de la Sociedad Chilena de Antropología. 28: 37-46.

Tuan, Y-F. 2008 (1977). Space and Place. The Perspective of Experience. University of Minnesota Press, Minneapolis-Londres.

Vecchi, R.; M. Colombo, R. Frontini, N. Mazzia y C. Bayón. 2007. "Nuevas evidencias arqueológicas en la localidad arqueológica El Guanaco (partido de San Cayetano, provincia de Buenos Aires)". Trabajo presentado en XVI Congreso Nacional de Arqueología Argentina, San Salvador de Jujuy, Argentina.

Zárate, M.; C. Bayón y N. Flegenheimer. 2009. “Tiempo, paisaje y ocupaciones 


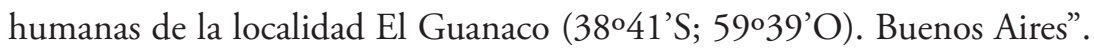
Trabajo presentado en IV Congreso Argentino de Cuaternario y Geomorfología, La Plata, Argentina. 